TRANSACTIONS OF THE

AMERICAN MATHEMATICAL SOCIETY

Volume 358, Number 10, October 2006, Pages 4367-4377

S 0002-9947(06)03844-X

Article electronically published on January 24, 2006

\title{
FRANKEL'S THEOREM IN THE SYMPLECTIC CATEGORY
}

\author{
MIN KYU KIM
}

\begin{abstract}
We prove that if an $(n-1)$-dimensional torus acts symplectically on a $2 n$-dimensional symplectic manifold, then the action has a fixed point if and only if the action is Hamiltonian. One may regard it as a symplectic version of Frankel's theorem which says that a Kähler circle action has a fixed point if and only if it is Hamiltonian. The case of $n=2$ is the well-known theorem by McDuff.
\end{abstract}

\section{INTRODUCTION}

One of the interesting problems in symplectic geometry is to find conditions to guarantee that a symplectic action must be Hamiltonian. It has a long historysince the beautiful theorem of Frankel which says that a Kähler circle action has a fixed point if and only if it is Hamiltonian $\mathrm{Fr}, \mathrm{O}$. For the history of the problem, see [M], MS, Section 1, Chapter 5]. In 1988, McDuff constructed a six-dimensional symplectic non-Hamiltonian circle action with fixed tori [M], which shows that Frankel's theorem is not true in the symplectic category. So, one may think that we need more symmetry for the existence of a fixed point to guarantee that a symplectic action must be Hamiltonian. In this report, we prove the following.

Theorem. Let an $(n-1)$-dimensional torus act symplectically on a $2 n$-dimensional symplectic manifold in an effective way. Then the action has a fixed point if and only if the action is Hamiltonian.

From McDuff's example and its products with copies of a two-dimensional sphere endowed with the usual rotation, we can see that the condition on the dimension of the acting torus is optimal to obtain the theorem. For $(n-1)$-dimensional torus actions on $2 n$-dimensional symplectic manifolds, see [KT1, [KT2].

To guarantee that a symplectic action must be Hamiltonian, we need some condition either on the manifold or on the action as noted in [MS, page 155]. For the former, Feldman shows that a symplectic circle action with nonempty fixed points on a manifold with the positive Todd genus is Hamiltonian [Fe. For the latter, Tolman and Weitsman show that a semifree symplectic circle action with nonempty isolated fixed points is Hamiltonian [TW]. It is also conceivable that a symplectic circle action with nonempty isolated fixed points must be Hamiltonian, but it is still open. In this report, we impose a restriction on the acting group itself. Recently, Sleewaegen reproved the theorem of this report under an additional assumption in Sl.

Received by the editors April 6, 2004 and, in revised form, August 11, 2004.

2000 Mathematics Subject Classification. Primary 53D05, 53D20; Secondary 55Q05, 57R19.

Key words and phrases. Symplectic geometry, symplectic action, Hamiltonian action.

(C)2006 American Mathematical Society

Reverts to public domain 28 years from publication 
In Section 2, we explain notation and define terminologies. Also, local behavior of generalized moment maps is investigated. The proof is given in Section 3. It is based on analysis of local behavior of generalized moment maps. Also, McDuff's paper [M, proof of Lemma 2] is used repeatedly and says that a symplectic circle action with a local extremum for a generalized moment map must be Hamiltonian.

\section{LOCAL DESCRIPTION OF GENERALIZED MOMENT MAPS}

It is well known that moment map images classify toric manifolds D. Similarly, in her beautiful paper [T] Tolman shows that moment map images (she calls them $\mathrm{x}$ rays) are very useful in dealing with six-dimensional Hamiltonian two-torus actions. In this section, we define the x-ray of an action and investigate local behavior of generalized moment maps through observing x-rays.

First, we explain notation. Let $\left(M^{2 n}, \omega\right)$ be a $2 n$-dimensional symplectic manifold. Let us fix a decomposition of the $(n-1)$-torus $T^{n-1}=S_{1}^{1} \times \cdots \times S_{n-1}^{1}$ where $S_{i}^{1}, i=1, \cdots, n-1$, are circle subgroups of $T^{n-1}$. We denote by $\mathfrak{t}$ the Lie algebra of $T^{n-1}$. Let $\Lambda_{0}$ be the kernel of the exponential map for $T^{n-1}$, i.e., the lattice of circle subgroups of $T^{n-1}$. We assume that $T^{n-1}$ acts symplectically on $M^{2 n}$ in an effective way with a nonempty fixed point set. We may assume that the symplectic form $\omega$ is integral, and hence admits a generalized moment map $M$, Lemma 1 . Let $\mathbb{R} / \mathbb{Z}$ valued functions $\mu_{i}, i=1, \cdots, n-1$, be generalized moment maps for the $S_{i}^{1}$ actions on $M^{2 n}$. We denote the range of generalized moment maps $\mu_{i}$ by $\mathbb{R} / \mathbb{Z}$ instead of $S^{1}$ to avoid confusion with groups acting on manifolds. Put $\mu=\left(\mu_{1}, \cdots, \mu_{n-1}\right)$. Locally, the range $(\mathbb{R} / \mathbb{Z})^{n-1}$ of the generalized moment map $\mu$ can be considered as $\mathfrak{t}^{*}$, and so every local result on moment maps including Local Convexity Theorem [GS is also true for generalized moment maps. For each $x$ in the fixed point set $M^{T^{n-1}}$, we denote by $\mathrm{T}_{x} M$ the tangent space of $M^{2 n}$ at $x$. We also denote by the same notation $\mathrm{T}_{x} M$ the linear isotropy representation of $T^{n-1}$ on the tangent space at $x$, and by $\alpha_{i, x}, i=1, \cdots, n$, the weights of $\mathrm{T}_{x} M$. The weights $\alpha_{i, x}$ can be regarded as elements of $\mathfrak{t}^{*}$ through differentiation.

For a symplectic circle action, it is known that the action is non-Hamiltonian if and only if each component of the fixed point set is not a local extremum for a generalized moment map of the action [M] proof of Lemma 2], and this is equivalent to saying that for each fixed point $x$ the cone generated by the weights $\alpha_{i, x}$ is equal to the whole $\left(L S^{1}\right)^{*}$ where $L S^{1}$ means the Lie algebra of $S^{1}$. Similarly, for a $2 n$ dimensional symplectic $(n-1)$-torus action, the cone generated by weights of the linear isotropy representation at each fixed point plays a key role in the report, and so the following notations are used.

Notation. For $\alpha_{i} \in \mathfrak{t}^{*}, i=1, \cdots, r$, let

$$
\begin{gathered}
S\left(\alpha_{1}, \cdots, \alpha_{r}\right)=\left\{s_{1} \alpha_{1}+\cdots+s_{r} \alpha_{r} \in \mathfrak{t}^{*} \mid s_{1}, \cdots, s_{r} \geq 0\right\}, \\
S^{\circ}\left(\alpha_{1}, \cdots, \alpha_{r}\right)=\left\{s_{1} \alpha_{1}+\cdots+s_{r} \alpha_{r} \in \mathfrak{t}^{*} \mid s_{1}, \cdots, s_{r}>0\right\} .
\end{gathered}
$$

Now, we define the x-ray of an action. Let $T_{1}, \cdots, T_{N}$ be the subgroups of $T^{n-1}$ which occur as stabilizers of points in $M^{2 n}$. Let $M_{i}$ be the set of points for which the stabilizer is $T_{i}$. We also denote such a set by $M_{T_{i}}$. By relabeling we can assume that $M_{i}$ 's are connected and the stabilizer of points in $M_{i}$ is $T_{i}$. Then, $M^{2 n}$ is a disjoint union of $M_{i}$ 's. Also, it is well known that $M_{i}$ is open dense in its closure and the closure is just a component of $M^{T_{i}}$. Let $\mathfrak{M}$ be the set of $M_{i}$ 's. Then, 
the $x$-ray of $\left(M^{2 n}, \omega, \mu\right)$ is defined as the set of $\mu\left(\overline{M_{i}}\right)$ 's for $M_{i} \in \mathfrak{M}$. Each image $\mu\left(\overline{M_{i}}\right)$ (resp. $\mu\left(M_{i}\right)$ ) is called an $m$-face (resp. an open $m$-face) of the x-ray if $T_{i}$ is $(n-1-m)$-dimensional. Our interest is in open $(n-2)$-faces of the x-ray which are of codimension one in $(\mathbb{R} / \mathbb{Z})^{n-1}$ by $[\mathrm{GS}$, Theorem 3.6]. See also Example 1 below.

The generalized moment map has a simple form in a neighborhood of each orbit. Let $x$ be a point of $M$ with the stabilizer $T_{x}^{n-1}$. The symplectic slice representation $V$ at $x$ is defined as the induced $T_{x}^{n-1}$ representation

$$
\left(\mathrm{T}_{x} T^{n-1} \cdot x\right)^{\omega} / \mathrm{T}_{x}\left(T^{n-1} \cdot x\right)
$$

where the superscript $\omega$ means the symplectic perpendicular with respect to the form $\omega$. Then, a neighborhood of the orbit $T^{n-1} \cdot x$ is equivariantly symplectomorphic to a neighborhood of

$$
E=T^{n-1} \times_{T_{x}^{n-1}}\left(\mathfrak{t}_{x}^{\circ} \times V\right)
$$

where $\mathfrak{t}_{x}^{\circ}$ is the annihilator of $\mathfrak{t}_{x}$ in $\mathfrak{t}^{*}$ and $T_{x}^{n-1}$ acts trivially on $\mathfrak{t}_{x}^{\circ}$. In the neighborhood, the generalized moment map is given by

$$
\mu([t, \eta, v])=\mu(x)+\eta+A^{*} \mu_{V}(v)
$$

where $A: \mathfrak{t} \rightarrow \mathfrak{t}_{x}$ is a projection, $A^{*}$ is dual to $A$, and $\mu_{V}: V \rightarrow \mathfrak{t}_{x}^{*}$ is the moment map for the symplectic slice representation $V$. For more details, see [LT, pp. 4209 4210].

Example 1. We visualize an open $(n-2)$-face $\mu\left(M_{i}\right)$. Assume that a point $x$ in $M_{i}$ has the one-dimensional stabilizer $T_{x}^{n-1}$. First, let us calculate stabilizers of points in $E$. It is easy to show that each point $[t, \eta, 0]$ in $E$ has the stabilizer $T_{x}^{n-1}$. But, to calculate stabilizers of other points we need to know the $T_{x}^{n-1}$ representation $V$. Note that the annihilator $\mathfrak{t}_{x}^{\circ}$ is $(n-2)$-dimensional, and hence the representation $V$ is complex two-dimensional. Since $T_{x}^{n-1}$ is abelian, the representation $V$ can be written as a sum of two complex one-dimensional subrepresentations

$$
V=V_{1} \oplus V_{2} \text {. }
$$

One of the following four cases holds. Let $H$ be the identity component of $T_{x}^{n-1}$. Note that $\mu([t, \eta, 0])=\mu(x)+\eta$ and the representation $V$ is nontrivial because the $T^{n-1}$ action is effective.

i. The subrepresentation $V_{1}$ is trivial. Then, the set of points with the stabilizer $T_{x}^{n-1}$ is the set $\left\{[t, \eta, v] \in E \mid v \in V_{1}\right\}$ because the $T_{x}^{n-1}$ representation $V_{2}$ is faithful. The image of the set under $\mu$ is contained in $\mu(x)+\mathfrak{t}_{x}^{\circ}$. The generalized moment map image is drawn in Figure 1(a).

ii. The subrepresentation $V_{1}$ is nontrivial but a trivial $H$ representation. Then, the set of points with the stabilizer $T_{x}^{n-1}$ is smaller than the set $\{[t, \eta, v] \in$ $\left.E \mid v \in V_{1}\right\}$. The image $\mu([t, \eta, v])$ for $v \in V_{1}$ is also contained in $\mu(x)+\mathfrak{t}_{x}^{\circ}$. Also, see Figure [1(a).

iii. Both $V_{1}$ and $V_{2}$ are nontrivial $H$ representations and $\mu_{V}(V) \neq \mathfrak{t}_{x}^{*}$. Then, the set of points with the stabilizer $T_{x}^{n-1}$ is the set $\{[t, \eta, 0] \in E\}$. The generalized moment map image is drawn in Figure 1(b).

iv. Both $V_{1}$ and $V_{2}$ are nontrivial $H$ representations and $\mu_{V}(V)=\mathfrak{t}_{x}^{*}$. Then, the set of points with the stabilizer $T_{x}^{n-1}$ is the set $\{[t, \eta, 0] \in E\}$. The generalized moment map image is drawn in Figure 1(c).

Note that in all four cases the open $(n-2)$-face $\mu\left(M_{i}\right)$ is locally of the form $\mu(y)+\mathfrak{t}_{x}^{\circ}$ for each $y \in M_{i}$. 


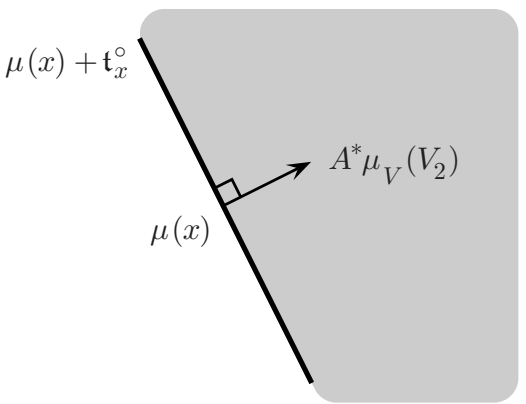

(a)

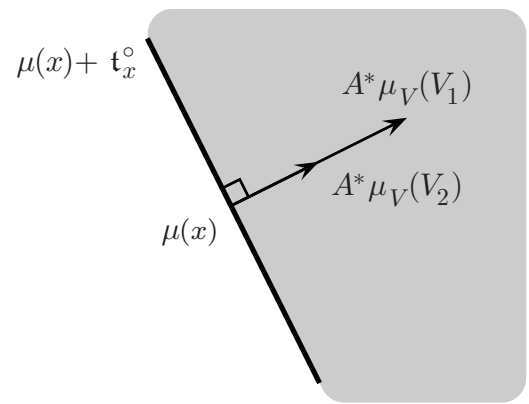

(b)

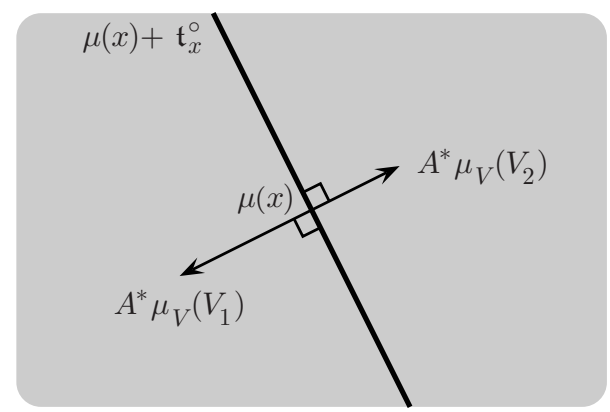

(c)

Figure 1. Generalized moment maps of Example 1

Now, we investigate local behavior of a generalized moment map near a fixed point through describing the x-ray. To do it, we need to calculate stabilizers near a fixed point. The $T^{n-1}$ representation $\mathrm{T}_{x} M$ for $x \in M^{T^{n-1}}$ can be expressed as

$$
\left(\alpha_{1} z_{1}, \cdots, \alpha_{n} z_{n}\right)
$$

and the moment map $\mu$ is written as

$$
\mu\left(z_{1}, \cdots, z_{n}\right)=\sum \frac{1}{2}\left|z_{i}\right|^{2} \alpha_{i},
$$

where $\alpha_{i}$ 's are the weights of $\mathrm{T}_{x} M$ and $z_{i} \in \mathbb{C}$. Then, we can easily verify that $\mu\left(\mathrm{T}_{x} M\right)=S\left(\alpha_{1}, \cdots, \alpha_{n}\right)$. For a while, we assume that the cone $S\left(\alpha_{1}, \cdots, \alpha_{n}\right)$ is equal to $\mathfrak{t}^{*}$. From this condition, any $(n-1)$ weights of $\alpha_{1}, \cdots, \alpha_{n}$ are independent in $\mathfrak{t}^{*}$. In other words, any $(n-1)$-dimensional matrix subrepresentation has a finite kernel. Also, we can regard an $(n-1)$-dimensional matrix subrepresentation as a homomorphism from $T^{n-1}$ to $T^{n-1^{\prime}}$ where the latter group $T^{n-1^{\prime}}$ acts diagonally on $\mathbb{C}^{n-1}$ by $\left(t_{1} z_{1}, \cdots, t_{n-1} z_{n-1}\right)$ for $t_{i} \in S^{1}$. From this, we obtain the following lemma. For $1 \leq i<j \leq n$, put

$$
W_{i, j}=\left\{\left(z_{1}, \cdots, z_{n}\right) \in \mathbb{C}^{n} \mid z_{i}=z_{j}=0, z_{k} \neq 0 \text { for } k \neq i, j\right\} .
$$

Lemma 1. For $n \geq 3$, any point in $W_{i, j}$ has the same stabilizer. The set of points in $\mathrm{T}_{x} M$ whose stabilizer is one-dimensional is the union of $W_{i, j}$ for $1 \leq i<j \leq n$.

Remark 1. Easily, we get $\mu\left(W_{i, j}\right)=S^{\circ}\left(\alpha_{1}, \cdots, \hat{\alpha_{i}}, \cdots, \hat{\alpha_{j}}, \cdots, \alpha_{n}\right)$ where the hat means a missing part. Let $H_{i, j}$ be the circle subgroup of $T^{n-1}$ such that $W_{i, j} \subset$ 


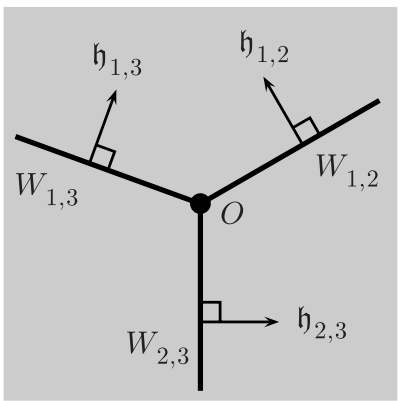

FiguRE 2. $W_{i, j}$ and $\mathfrak{h}_{i, j}$ of Remark 1

$\left(\mathrm{T}_{x} M\right)^{H_{i, j}}$, i.e., each $H_{i, j}$ is the identity component of the stabilizer of $W_{i, j}$. Since any $(n-1)$ weights of $\alpha_{1}, \cdots, \alpha_{n}$ are linearly independent, for $\{i, j\} \neq\{k, l\}$ the images $\mu\left(W_{i, j}\right)$ and $\mu\left(W_{k, l}\right)$ span different vector spaces, respectively. Thus, by Example 1 or [GS, Theorem 3.6], we obtain $H_{i, j} \neq H_{k, l}$ for $\{i, j\} \neq\{k, l\}$. Also, see Figure 2.

Remark 2. Let $M_{i}$ be an element of $\mathfrak{M}$ with a one-dimensional stabilizer $T_{i}$, and let $H$ be the identity component of $T_{i}$. Assume that the closure $\overline{M_{i}}$ contains a fixed point $x \in M^{T^{n-1}}$ such that $S\left(\alpha_{1, x}, \cdots, \alpha_{n, x}\right)=\mathfrak{t}^{*}$. By Lemma1, $\operatorname{dim} M_{i}=2(n-2)$. Forgetting the whole group $T^{n-1}$ action on $M^{2 n}$, we only consider the $H$ action on $M^{2 n}$. Since $H$ fixes $\overline{M_{i}},(n-2)$ weights of $\left.\alpha_{1, x}\right|_{\mathfrak{h}}, \cdots,\left.\alpha_{n, x}\right|_{\mathfrak{h}}$ are zero as elements of $\mathfrak{h}^{*}$. Since $S\left(\alpha_{1, x}, \cdots, \alpha_{n, x}\right)=\mathfrak{t}^{*}$ implies $S\left(\left.\alpha_{1, x}\right|_{\mathfrak{h}}, \cdots,\left.\alpha_{n, x}\right|_{\mathfrak{h}}\right)=\mathfrak{h}^{*}$, the remaining two nonzero weights have different signs in $\mathfrak{h}^{*}$. Therefore, the $H$ representation on the fiber at $x$ of the normal bundle of $\overline{M_{i}}$ can be expressed as $\left(t^{d} z_{1}, t^{-d^{\prime}} z_{2}\right)$ for $t \in H$ where $d$ and $d^{\prime}$ are positive integers. Also, since $H$ fixes $\overline{M_{i}}$, the $H$ representation on each fiber of the normal bundle is all the same, and we can show that it is $V$ in Example 1, This gives a description of a generalized moment map near $M_{i}$ by Example 1-iv. Moreover, these arguments also apply to the case $S\left(\alpha_{1, x}, \cdots, \alpha_{n, x}\right) \neq \mathfrak{t}^{*}$. From this, one may say that the cone $S\left(\alpha_{1, x}, \cdots, \alpha_{n, x}\right)$ of a fixed point $x$ in $\overline{M_{i}}$ determines the generalized moment map image near $\overline{M_{i}}$.

Next, we investigate the image (not the x-ray) of $\mu$ for the $T^{n-1}$ representation $\mathrm{T}_{x} M$ when $x \in M^{T^{n-1}}$ and $S\left(\alpha_{1}, \cdots, \alpha_{n}\right) \neq \mathfrak{t}^{*}$. For $v \in \mathfrak{t}$, we denote by $v^{\circ}$ the annihilator of $v$ in $\mathfrak{t}^{*}$ with respect to the pairing between $\mathfrak{t}$ and $\mathfrak{t}^{*}$.

Lemma 2. Let $\alpha_{1}, \cdots, \alpha_{n}$ be the weights of a $T^{n-1}$ representation on $\mathbb{C}^{n}$. Assume that $S\left(\alpha_{1}, \cdots, \alpha_{n}\right) \neq \mathfrak{t}^{*}$. Then, the cone $S\left(\alpha_{1}, \cdots, \alpha_{n}\right)$ satisfies either

i. it contains no nontrivial vector space, and there is a vector $v_{0}$ in $\Lambda_{0}$ such that $v_{0}^{\circ} \cap S\left(\alpha_{1}, \cdots, \alpha_{n}\right)=0$, or

ii. it contains a nontrivial vector space, and the cone is homeomorphic to $\mathbb{R}^{l} \times$ $\left(\mathbb{R}_{>0}^{1}\right)^{n-1-l}$ for some $l \in \mathbb{N}$. Also, there is a vector $v_{0}$ in $\Lambda_{0}$ such that $v_{0}^{\circ} \cap S\left(\alpha_{1}, \cdots, \alpha_{n}\right)$ is an l-dimensional vector space.

Proof. For a point $x$ having the identity as $T_{x}^{n-1}$, the moment map is a submersion near $x$ by the simple form of $\mu$. Therefore, weights $\alpha_{1}, \cdots, \alpha_{n}$ span $\mathfrak{t}^{*}$ as a vector space because the moment map image is equal to $S\left(\alpha_{1}, \cdots, \alpha_{n}\right)$. From this, we can find $(n-1)$ independent weights (say $\alpha_{1}, \cdots, \alpha_{n-1}$ ). Put $\alpha_{n}=\sum_{i=1}^{n-1} d_{i} \alpha_{i}$. By 
a suitable linear transformation, we may assume that each $\alpha_{i}$ is the usual basis element $e_{i}$ of $\mathbb{R}^{n-1}$ for $i=1, \cdots, n-1$. In the coordinates, $\alpha_{n}=\left(d_{1}, \cdots, d_{n-1}\right)$.

Now, assume that $S\left(\alpha_{1}, \cdots, \alpha_{n}\right)$ contains no nontrivial vector space. Then, $\alpha_{n}$ is the zero vector or at least one of the $d_{i}$ 's is positive. If $\alpha_{n}$ is the zero vector, then take $v_{0}=(-1, \cdots,-1)$ and we are done. Otherwise, we can find a vector $v_{0}=\left(a_{1}, \cdots, a_{n-1}\right)$ with negative integers $a_{i}$ such that all the pairings $\left\langle v_{0}, \alpha_{i}\right\rangle$ are negative. Then, $v_{0}^{\circ} \cap S\left(\alpha_{1}, \cdots, \alpha_{n}\right)=0$. If $v_{0}$ is not contained in $\Lambda_{0}$, then we only have to multiply $v_{0}$ by a big natural number to obtain a wanted vector in $\Lambda_{0}$.

Next, assume that $S\left(\alpha_{1}, \cdots, \alpha_{n}\right)$ contains a nontrivial vector space. This implies that each $d_{i}$ is nonpositive. Let $l$ be the number of negative $d_{i}$ 's (say $d_{1}, \cdots, d_{l}$ ). Then, $S\left(\alpha_{1}, \cdots, \alpha_{n}\right)$ is equal to $\mathbb{R}^{l} \times\left(\mathbb{R}_{\geq 0}^{1}\right)^{n-1-l}$. Put $v_{0}=\left(a_{1}, \cdots, a_{n-1}\right)$ with $a_{1}, \cdots, a_{l}=0$ and $a_{l+1}, \cdots, a_{n-1}=-1$. Then, $v_{0}^{\circ} \cap S\left(\alpha_{1}, \cdots, \alpha_{n}\right)=\mathbb{R}^{l} \times 0$.

Example 2. Figure 3 illustrates Lemma 2 when $n=3$ and $\alpha_{3} \neq 0$. The case of $\alpha_{3}=0$ is the same with Figure [3)(a) except $\alpha_{3}=0$.

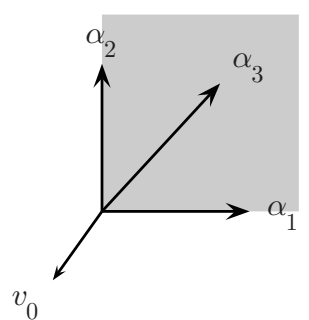

(a)

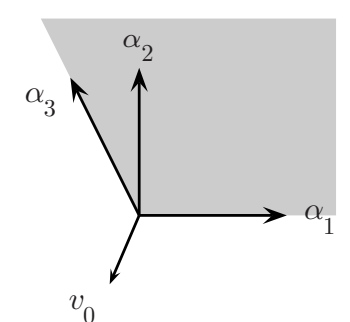

(b)

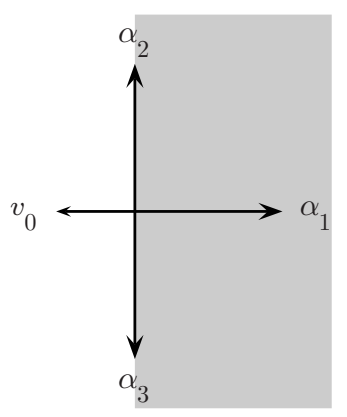

(c)

Figure 3. Three cases of $S\left(\alpha_{1}, \alpha_{2}, \alpha_{3}\right)$ when $\alpha_{3} \neq 0$

\section{ProOF}

First, we prove the following lemma about an equivalent condition to guarantee that a symplectic action must be Hamiltonian.

Lemma 3. Let an m-dimensional torus $T^{m}$ act on $\left(M^{2 n}, \omega\right)$. Then the action is Hamiltonian if and only if there exists a component $F$ of $M^{T^{m}}$ such that the map $\pi_{1}(F) \rightarrow \pi_{1}\left(M^{2 n}\right)$ is surjective. 
Proof. Assume that the action is Hamiltonian. Let $F$ be $\mu^{-1}(p)$ where $\mu$ is a moment map for the action and the point $p$ is an extreme point (or a vertex) of the image of $\mu$. For an extreme point, see $[\underline{\mathrm{R}}$, Chapter 3]. Then, $F$ is a component of $M^{T^{m}}$ and connected by the Convexity Theorem [A], [GS]. By [Fu, p. 14 (13)], there exists a vector $v_{0} \in \Lambda_{0} \subset \mathfrak{t}$ such that $\left(p+v_{0}^{\circ}\right) \cap \mu\left(M^{2 n}\right)=p$ since $p$ is an extreme point of $\mu\left(M^{2 n}\right)$. Let $H$ be the circle subgroup of $T^{m}$ corresponding to $v_{0}$. Then, a moment map $\mu_{H}$ for the $H$ action attains its extremum at $F$, and hence $F$ is also a component of $M^{H}$. By $\left[\mathrm{L}\right.$, the fundamental groups $\pi_{1}\left(M^{2 n}\right), \pi_{1}\left(M_{\min }\right), \pi_{1}\left(M_{\max }\right)$ are all the same where $M_{\min }$ and $M_{\max }$ mean the preimages of the minimum and maximum under $\mu_{H}$, respectively. Thus, $\pi_{1}(F) \rightarrow \pi_{1}\left(M^{2 n}\right)$ is surjective.

We prove the converse. Assume that for a circle subgroup $H$ of $T^{m}$, the $H$ action is non-Hamiltonian. Then, there exists a loop in $M^{2 n}$ such that its image under a generalized moment map $\mu_{H}$ for the $H$ action is nontrivial in $\pi_{1}(\mathbb{R} / \mathbb{Z})$. But, the loop cannot be deformed into a component $F$ of $M^{T^{m}}$ because $\mu_{H}(F)$ is a point in $\mathbb{R} / \mathbb{Z}$.

We prove the theorem by induction on $n$. The case of $n=2$ is McDuff's theorem [M]. Suppose that the theorem is true when the dimension of the manifold $M$ is less than $2 n$. The situation consists of the following two cases.

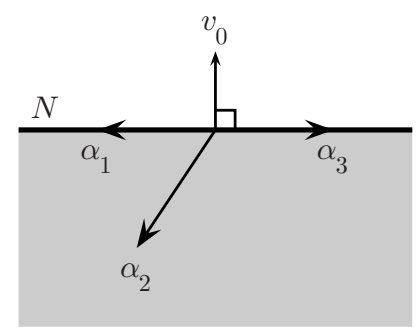

Figure 4. Proof of Case 1

Case 1: There exists a fixed point $x$ such that $S\left(\alpha_{1, x}, \cdots, \alpha_{n, x}\right) \neq \mathfrak{t}^{*}$.

By Lemma 2, there exists a vector $v_{0} \in \Lambda_{0} \subset \mathfrak{t}$ such that the pairing $\left\langle v_{0}, S\left(\alpha_{1, x}, \cdots, \alpha_{n, x}\right)\right\rangle$ is nonpositive and $v_{0}^{\circ} \cap S\left(\alpha_{1, x}, \cdots, \alpha_{n, x}\right)$, say $Z$, is an $l$ dimensional vector space for some $0 \leq l \leq n-2$. We denote by $H$ the circle subgroup of $T^{n-1}$ corresponding to $v_{0}$. Then, a generalized moment map $\mu_{H}$ of the $H$ action attains its local maximum, and hence the $H$ action is Hamiltonian as noted in the proof of [M, Lemma 2]. So, we can assume that $\mu_{H}$ is an $\mathbb{R}$-valued moment map with the maximum 0 . If we put $N=\mu_{H}^{-1}(0)$, then $\pi_{1}(N) \rightarrow \pi_{1}\left(M^{2 n}\right)$ is surjective by $\left[\mathrm{L}\right.$. If $l=0$ and all $\alpha_{i, x}$ are nonzero, then $N=\{x\}$ and we obtain the proof by Lemma 3. Similarly, if $l=0$ and one of the $\alpha_{i, x}$ 's is zero, then $N$ is a two-surface fixed by $T^{n-1}$ and the proof is obtained in the same way.

Now, assume that $l \geq 1$. Then, $\operatorname{dim} N=2(l+1)$ by the proof of Lemma 2 Also, $N$ is fixed by the $(n-1-l)$-dimensional subtorus $\exp \left(Z^{\circ}\right)$ generated by $Z^{\circ}$. There is an $l$-dimensional subtorus of $T^{n-1}$ denoted by $T^{l}$ whose Lie algebra and $Z^{\circ}$ span $\mathfrak{t}$, i.e., $T^{n-1}=T^{l} \cdot \exp \left(Z^{\circ}\right)$. The subtorus $T^{l}$ acts symplectically on $N$ with a nonempty fixed point set containing $x$ because $x \in M^{T^{n-1}}$. Thus, the $T^{l}$ action on $N$ is Hamiltonian by the induction hypothesis. Therefore, any loop in $N$ can be deformed into a component $F$ of $N^{T^{l}}=N^{T^{n-1}}$, i.e., $\pi_{1}(F) \rightarrow \pi_{1}(N)$ is surjective. 
Since we already have the surjection $\pi_{1}(N) \rightarrow \pi_{1}\left(M^{2 n}\right)$, the proof is obtained again by Lemma 3 .

Case 2: For each fixed point $x$, we have $S\left(\alpha_{1, x}, \cdots, \alpha_{n, x}\right)=\mathfrak{t}^{*}$.

To obtain the proof, we need to prove the following lemma. Let $M_{i}$ be an element of $\mathfrak{M}$ with a one-dimensional stabilizer $T_{i}$. We prove that if the generalized moment map $\mu$ behaves like either Figure 1(a) or 1(b) near a point in $M_{i}$, then the closure $\overline{M_{i}}$ contains a point in $M^{T^{n-1}}$. But, this cannot happen under the assumption of Case 2 by Remark 2 .

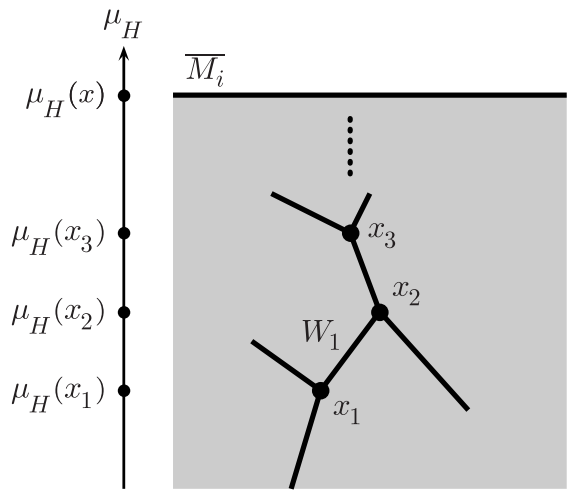

Figure 5. Proof of Lemma 4

Lemma 4. Let $x$ be a point in $M_{i}$ with a one-dimensional stabilizer $T_{i}$. If $\mu_{V}(V) \neq$ $\mathfrak{t}_{x}^{*}$, then $\overline{M_{i}}$ contains a point in $M^{T^{n-1}}$ where $V$ is the symplectic slice representation at $x$ and $\mu_{V}$ is its moment map.

Proof. Assume that $\overline{M_{i}}$ does not contain any point in $M^{T^{n-1}}$. Let $H$ be the identity component of $T_{i}=T_{x}^{n-1}$. Let $\mu_{H}$ be the generalized moment map for the $H$ action given by

$$
\mu_{H}([t, \eta, v])=\mu(x)+\mu_{V}(v)
$$

near the orbit $T^{n-1} \cdot x$. Since $H$ fixes $\overline{M_{i}}$ and $\mu_{V}(V) \neq \mathfrak{t}_{x}^{*}$, the map $\mu_{H}$ has a local extremum at $\overline{M_{i}}$, say maximum $\mu_{H}(x)$, and hence the $H$ action is Hamiltonian. Therefore, we can assume that the function $\mu_{H}$ is $\mathbb{R}$-valued.

Let $x_{1} \in M^{T^{n-1}}$. Then, by the assumption the weights of $\mathrm{T}_{x_{1}} M$ satisfy $S\left(\alpha_{1, x_{1}}, \cdots, \alpha_{n, x_{1}}\right)=\mathfrak{t}^{*}$, and this implies $\mu_{H}\left(x_{1}\right)<\mu_{H}(x)$ because $x_{1}$ is not a local extremum for $\mu_{H}$. Since $S\left(\alpha_{1, x_{1}}, \cdots, \alpha_{n, x_{1}}\right)=\mathfrak{t}^{*}$, there is a $W_{k, l}$ of Lemma 1 in $\mathrm{T}_{x_{1}} M$ such that $x_{1} \in \bar{W}_{k, l}$ and $\mu_{H}\left(x_{1}\right)<\mu_{H}(y)$ for some $y \in \bar{W}_{k, l}$. Let $T_{i_{1}}$ be the stabilizer of $W_{k, l}$, and let $W_{1}$ be the component of $M_{T_{i_{1}}}$ such that $\bar{W}_{1}$ contains $x_{1}$, i.e., $\bar{W}_{k, l}$ is a linear approximation of $\bar{W}_{1}$ near $x_{1}$. Then, the induced $T^{n-1} / T_{i_{1}}$ action on $\bar{W}_{1}$ is effective and Hamiltonian by the induction hypothesis. Also, $\bar{W}_{1}$ has another fixed point $x_{2}$ for the $T^{n-1}$ action such that $\mu_{H}\left(x_{1}\right)<\mu_{H}\left(x_{2}\right)$ because $\mu_{H}\left(x_{1}\right)$ is not the maximum of $\mu_{H}\left(\bar{W}_{1}\right)$. Since $\mu_{H}\left(x_{2}\right)<\mu_{H}(x)$, we can repeat this process infinite times to obtain a sequence $\left\{x_{i}\right\}_{i \in \mathbb{N}} \subset M^{T^{n-1}}$ such that

$$
\mu_{H}\left(x_{1}\right)<\mu_{H}\left(x_{2}\right)<\mu_{H}\left(x_{3}\right)<\cdots .
$$

This is a contradiction. 


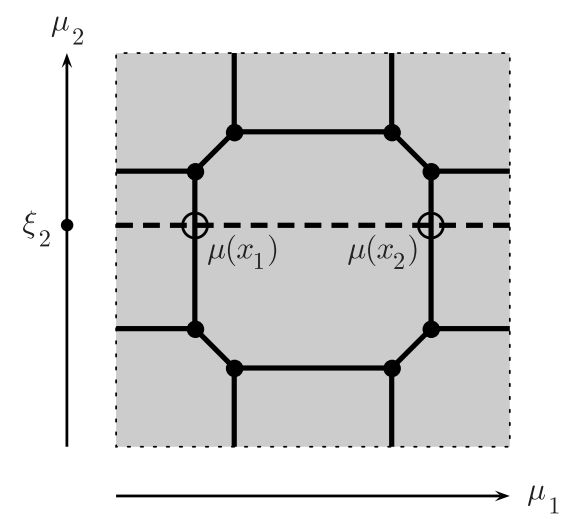

Figure 6. Illustration of the idea of proof

Before we start the proof of Case 2, we will illustrate the idea of proof with the following example of the case $n=3$. Assume that there exists a six-dimensional two-torus action whose x-ray is Figure [6. Note that $S\left(\alpha_{1, x}, \alpha_{2, x}, \alpha_{3, x}\right)=\mathfrak{t}^{*}$ for each $x \in M^{T^{2}}$. Let us fix a decomposition of the two-torus $T^{2}=S_{1}^{1} \times S_{2}^{1}$, and let $\mathbb{R} / \mathbb{Z}$-valued functions $\mu_{i}$ be generalized moment maps for the $S_{i}^{1}$ actions. Put $\mu=\left(\mu_{1}, \mu_{2}\right)$. The gray square is the range $\mathbb{R} / \mathbb{Z} \times \mathbb{R} / \mathbb{Z}$ of $\mu$. Each black dot is the image of a fixed point, and each thick line is the image of an open one-face. Let $\xi_{2} \in \mathbb{R} / \mathbb{Z}$ be a regular value for $\mu_{2}$. Then, the thick dashed line becomes the x-ray of the induced symplectic $T^{2} / S_{2}^{1}$ action on the four-dimensional orbifold $\mu_{2}^{-1}\left(\xi_{2}\right) / S_{2}^{1}$. The induced action has two isolated fixed points which are orbits of $x_{1}$ and $x_{2}$. Also, the induced action has the generalized moment map induced from $\mu_{1}$, and the induced generalized moment map cannot be an $\mathbb{R}$-valued function because two fixed points are not locally extremal. But, it is impossible by the same proof of [M. Lemma 3] in the orbifold setting. Thus, there is no action with Figure [6 as an x-ray.

Now, we start the proof. We can choose a generic regular value $\xi_{i} \in \mathbb{R} / \mathbb{Z}$ for each $\mu_{i}, i=2, \cdots, n-1$, such that in the range of $\mu$, the subset $(\mathbb{R} / \mathbb{Z}) \times \xi_{2} \times \cdots \times \xi_{n-1}$ meets each open $m$-face transversely for $m=1, \cdots, n-2$ and there is at least one open $(n-2)$-face intersecting the subset. Transversality guarantees that the subset $(\mathbb{R} / \mathbb{Z}) \times \xi_{2} \times \cdots \times \xi_{n-1}$ does not meet any open $m$-face for $m \leq n-3$, and this is why our interest is mainly in (open) $(n-2)$-faces.

Put $\xi=\left(\xi_{2}, \cdots, \xi_{n-1}\right)$. Also, we put $V_{\xi}=\left(\mu_{2}, \cdots, \mu_{n-1}\right)^{-1}(\xi)$. Then, the orbit space $B_{\xi}=V_{\xi} /\left(S_{2}^{1} \times \cdots \times S_{n-1}^{1}\right)$ is a four-dimensional orbifold naturally endowed with a symplectic form. For orbifolds see $[\mathrm{Sa}$, and for symplectic actions on orbifolds see [LT]. Denote $T^{n-1} /\left(S_{2}^{1} \times \cdots \times S_{n-1}^{1}\right)$ by $H$. If we restrict the generalized moment map $\mu_{1}$ to $V_{\xi}$, then we obtain the induced generalized moment map $\mu_{H}: B_{\xi} \rightarrow \mathbb{R} / \mathbb{Z}$ for the induced symplectic $H$ action on $B_{\xi}$. Also, the $H$ action on $B_{\xi}$ is effective for a generic $\xi$. However, we do not know whether $\mu_{H}$ can be regarded as an $\mathbb{R}$-valued function.

Let $\pi: V_{\xi} \rightarrow B_{\xi}$ be the orbit map. For $x \in V_{\xi}$, the point $\pi(x)$ is an $H$ fixed point if and only if the orbit $T^{n-1} \cdot x$ is $(n-2)$-dimensional because $S_{2}^{1} \times \cdots \times S_{n-1}^{1}$ acts almost freely on $V_{\xi}$, i.e., stabilizers are all finite groups, and hence $H \cdot x \subset$ 
$\left(S_{2}^{1} \times \cdots \times S_{n}^{1}\right) \cdot x$. This is also equivalent to say that the stabilizer of $x$ is onedimensional. Since $(\mathbb{R} / \mathbb{Z}) \times \xi$ meets at least one open $(n-2)$-face and an open $(n-2)$-face is an image of some $M_{i} \in \mathfrak{M}$ with a one-dimensional $T_{i}, B_{\xi}$ has at least one fixed point.

Let $\pi(x) \in B_{\xi}$ be a fixed point of the $H$ action. The orbit space $B_{\xi}$ need not be connected because $V_{\xi}$ need not be connected. So, we denote the component of $B_{\xi}$ containing $\pi(x)$ by the same notation $B_{\xi}$. From Remark 2 and Lemma 4 , the x-ray of the $T^{n-1}$ action on $M^{2 n}$ is locally of the form of Figure 1(c) near $\mu(x)$. Also, see Figure 7 Therefore, we can conclude that each fixed point in $B_{\xi}$ is an isolated critical point because it is not extremal for $\mu_{H}$.

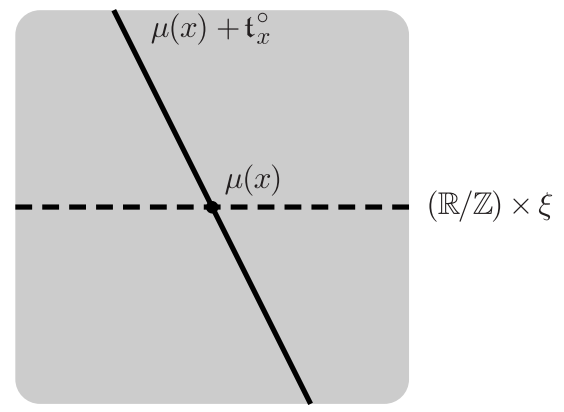

Figure 7. Proof of Case 2

In summary, we obtain a symplectic circle action on a four-dimensional symplectic orbifold with nonempty isolated fixed points which are nonextremal for a generalized moment map. McDuff shows that such an action does not exist if the orbifold is a smooth manifold [M, Lemma 3]. And, it is easy to see that her proof can be transferred almost literally to our case, and we obtain a contradiction. Therefore, Case 2 cannot happen.

As a corollary of the above proof, we obtain the following. We define the dimension of the fixed point set of an action as the maximum of dimensions of components of the fixed point set of the action.

Corollary. Let $T^{m}$ act symplectically on $\left(M^{2 n}, \omega\right)$ with a nonempty $M^{T^{m}}$ in an effective way. If $\operatorname{dim} M^{T^{m}} \geq 2(n-m)$, then the action is Hamiltonian. In particular, if $m=n$, then the action has a fixed point if and only if it is Hamiltonian.

Proof. Let $F$ be a component of the fixed point set such that $\operatorname{dim} F=\operatorname{dim} M^{T^{m}}$. By the assumption, $\operatorname{dim} F \geq(2 n-2 m)$. Then at any point $x \in F$, there $\operatorname{are} n-\operatorname{dim} F / 2$ nonzero weights of $\mathrm{T}_{x} M$. Since $m \geq n-\operatorname{dim} F / 2$ and the weights span $\mathfrak{t}^{*}$ as a vector space, the number $n-\operatorname{dim} F / 2$ must be equal to $m$. The nonzero weights are linearly independent in $\mathfrak{t}^{*}$, and hence $S\left(\alpha_{1, x}, \cdots, \alpha_{n, x}\right)$ is strictly convex, i.e., it contains no nontrivial vector space. By the same arguments of the proof of the Case 1, we obtain the desired proof.

\section{ACKNOWLEDGMENTS}

I would like to give special thanks to Dong Youp Suh for bringing the paper of Tolman and Weitsman to my attention. It was the beginning of this research. 
I would like to thank Jin-Whan Yim for many interesting discussions. I also would like to thank Seung-Taik Oh and Myung-Jun Choi for teaching me how to draw pictures.

\section{REFERENCES}

[A] M. F. Atiyah, Convexity and commuting Hamiltonians, Bull. London Math. Soc. 14 (1982), no. 1, 1-15. MR0642416 (83e:53037)

[D] T. Delzant, Hamiltoniens periodiques et images convexes de l'application moment, Bull. Soc. Math. France 116 (1988), 315-339. MR0984900 (90b:58069)

[Fe] K. E. Feldman, Hirzebruch genera of manifolds supporting a Hamiltonian circle action, Russian Math. Surveys 56 (2001), no. 5, 978-979. MR.1892568 (2003a:57062)

[Fr] T. Frankel, Fixed points on Kähler manifolds, Ann. Math. 70 (1959), 1-8. MR0131883 (24:A1730)

[Fu] W. Fulton, Introduction to Toric Varieties, Ann. Math. Studies 131, Princeton University Press, 1993. MR1234037 (94g:14028)

[GS] V. Guillemin, S. Sternberg, Convexity properties of the moment mapping, Invent. Math. 67 (1982), no. 3, 491-513. MR0664117 (83m:58037)

[KT1] Y. Karshon, S. Tolman, Centered complexity one Hamiltonian torus actions, Trans. Amer. Math. Soc. 353 (2001), no. 12, 4831-4861. MR.1852084 (2002g:53145)

[KT2] Y. Karshon, S. Tolman, Complete invariants for Hamiltonian torus actions with two dimensional quotients, J. Symplectic Geom. 2 (2003), no. 1, 25-82. MR2128388

[LT] E. Lerman, S. Tolman, Hamiltonian torus actions on symplectic orbifolds and toric varieties, Trans. Amer. Math. Soc. 349 (1997), no. 10, 4201-4230. MR.1401525 (98a:57043)

[L] H. Li, $\pi_{1}$ of Hamiltonian $S^{1}$ manifolds, Proc. Amer. Math. Soc. 131, no. 11, 3579-3582. MR.1991771 (2004b:53145)

$[\mathrm{M}] \quad$ D. McDuff, The moment map for circle actions on symplectic manifolds, J. Geom. Phys. 5 (1988), no. 2, 149-160. MR1029424 (91c:58042)

[MS] D. McDuff, D. Salamon, Introduction to Symplectic Topology, 2nd ed., Oxford University Press, 1998. MR1698616 (2000g:53098)

[O] K. Ono, Equivariant projective imbedding theorem for symplectic manifolds, J. Fac. Sci. Univ. Tokyo Sect. IA Math. (2) 35 (1988), no. 2, 381-392. MR0945884 (89k:58103)

[R] W. Rudin, Functional Analysis, 2nd ed., McGraw-Hill, 1991. MR1157815 (92k:46001)

[Sa] I. Satake, On a generalization of the notion of manifold, Proc. Nat. Acad. Sci. U.S.A., 42 (1956), 359-363. MR0079769 (18:144a)

[Sl] P. Sleewaegen, On generalized moment maps for symplectic compact group actions, math. $\mathrm{SG} / 0304487$.

[SL] R. Sjamaar, E. Lerman, Stratified symplectic spaces and reduction, Ann. Math. 134 (1991), no. 2, 375-422. MR1127479 (92g:58036)

[T] S. Tolman, Examples of non-Kähler Hamiltonian torus actions, Invent. Math. 131 (1998), no. 2, 299-310. MR1608575 (2000d:53129)

[TW] S. Tolman, J. Weitsman, On semifree symplectic circle actions with isolated fixed points, Topology 39 (2000), no. 2, 299-309. MR.1722020 (2000k:53074)

Department of Mathematics, Korea Advanced Institute of Science and Technology, 373-1, Kusong-Dong, Yusong-Gu, TAejon, 305-701, Korea

E-mail address: minkyu@kaist.ac.kr

Current address: School of Math, Korea Institute for Advanced Study, Cheongnyangni 2-dong, Dongdaemun-gu, Seoul, 130-722, Republic of Korea

E-mail address: mkkim@kias.re.kr 